\section{CASE REPORT}

'Hospital Erasto Gaertner, Curitiba, PR, Brasil

Financial support: None. Conflicts of interest: No conflicts of interest declared concerning the publication of this article. Submitted: December 15, 2016. Accepted: July 13, 2017.

The study was carried out at Hospital Erasto Gaertner, Curitiba, PR, Brazil.

Copyright Pedruzzi et al. This is an Open Access article distributed under the terms of the Creative Commons Attribution License, which permits unrestricted use, distribution, and reproduction in any medium, provided the original work is properly cited.

\title{
Kimura's disease
}

\author{
Paola Andrea Galbiatti Pedruzzi ${ }^{1 *}$, Benedito Valdecir de Oliveira1, \\ Jacobson Correia Leite de Britto1, Roberta Targa Stramandinoli', \\ Ana Helena Willrich ${ }^{1}$, Juliana Elizabeth Jung ${ }^{1}$
}

\begin{abstract}
A 72-years-old man presents with a bilateral mass in the retroauricular region, diagnosed as Kimura disease. Kimura disease is a chronic disorder of unknown origin, common in East Asia but rare in other world regions. The clinical presentation of this disease is the triad of lymphadenopathy in the head and neck, peripheral blood eosinophilia and increased levels of immunoglobulin E. Treatment includes corticosteroids, chemotherapy, radiotherapy, and surgery.
\end{abstract}

Keywords: angiolymphoid hyperplasia with eosinophilia; eosinophilia; lymph nodes.

\section{Introduction}

Kimura's disease (KD) was first described in 1937 in the Chinese literature by Kim and Szeto' as "hyperplastic eosinophilic lymphogranuloma", known as KD after publication by Kimura et al. ${ }^{2}$ in 1948. It is a rare inflammatory disorder and it has an unknown cause, most commonly seen in the East ${ }^{3,4}$. There were just over 120 cases published in the literature until 2000 worldwide.

\section{Case report}

The case report was about a 72-year-old male patient, with parents born in Japan, admitted to the Erasto Gaertner Hospital, Curitiba, Paraná, Brazil in 2010, reporting a slow-growing bilateral retroauricular mass for 12 months. He had generalized pruritus five years ago (Figure 1). He is a former smoker (20 cigarettes per day for 48 years), reported drinking 2 glasses of beer on weekends for 48 years and exposure to pesticide benzene hexachloride (BHC) for 4 years. He had hypertension and diabetes mellitus as comorbidities. He did not present a family history of neoplasias. He said he lived in Japan for 14 years while working in the automobile industry. The patient was in general preserved state, without weight loss, with cervical mass in the left retroauricular region of $9 \times 7 \mathrm{~cm}$ in intimate contact with the inferior pole of parotid and right retroauricular mass of $4 \times 2 \mathrm{~cm}$. Absence of other cervical, axillary and inguinal lymphadenopathy. The hemogram showed leukocytes of 9790/ $\mathrm{mm}^{3}$, eosinophils 2252 (23\%), creatinine $1.14 \mathrm{mg} / \mathrm{dl}$, partial urine examination demonstrated absence of proteinuria and hypergammaglobulinemia $\mathrm{E}$. The fine needle aspiration showed the presence of lymphocytes without atypia, and the excisional biopsy of the retroauricular mass showed follicular hyperplasia demonstrating prominent germinative centers, associated with a marked inflammatory infiltrate of nodular and diffuse pattern, composed of lymphocytes and large amount of 
eosinophils (Figure 2A). The histopathological findings were compatible with Kimura's disease. Complementary immunohistochemical examination was performed, and the lymphocyte positivity for CD20 in the follicles showed follicular hyperplasia (Figure 2B). CD3 was positive in rare lymphocytes and CD34 demonstrated regular and well-defined blood vessels, which contributes to the exclusion of the differential diagnosis of angiolymphoid hyperplasia with eosinophilia. Cervical computed tomography (CT) revealed thickening of the soft and subcutaneous parts of the occipital, left retroauricular, irregular, poorly delimited lesions with the posterior portions of the parotid and multiple intraparotid lymph nodes, deep cervical and retroauricular. Magnetic resonance imaging (MRI) showed a subcutaneous lesion of the left retroauricular region, adjacent to mastoid with homogeneous enhancement after administration of intravenous contrast, measuring about $6.7 \times 5.8 \times 1.9 \mathrm{~cm}$. The lesion has an intimate relationship with the posterior region of the left parotid gland (Figure 3). The patient was submitted to resection of the left retroauricular lesion, with operative findings of compression of the external auditory canal, peripar-tidal lymph node enlargement, without a cleavage plane with the parotid gland that was partially resected, free surgical margins. The follow-up time is 90 months, with no signs of disease.

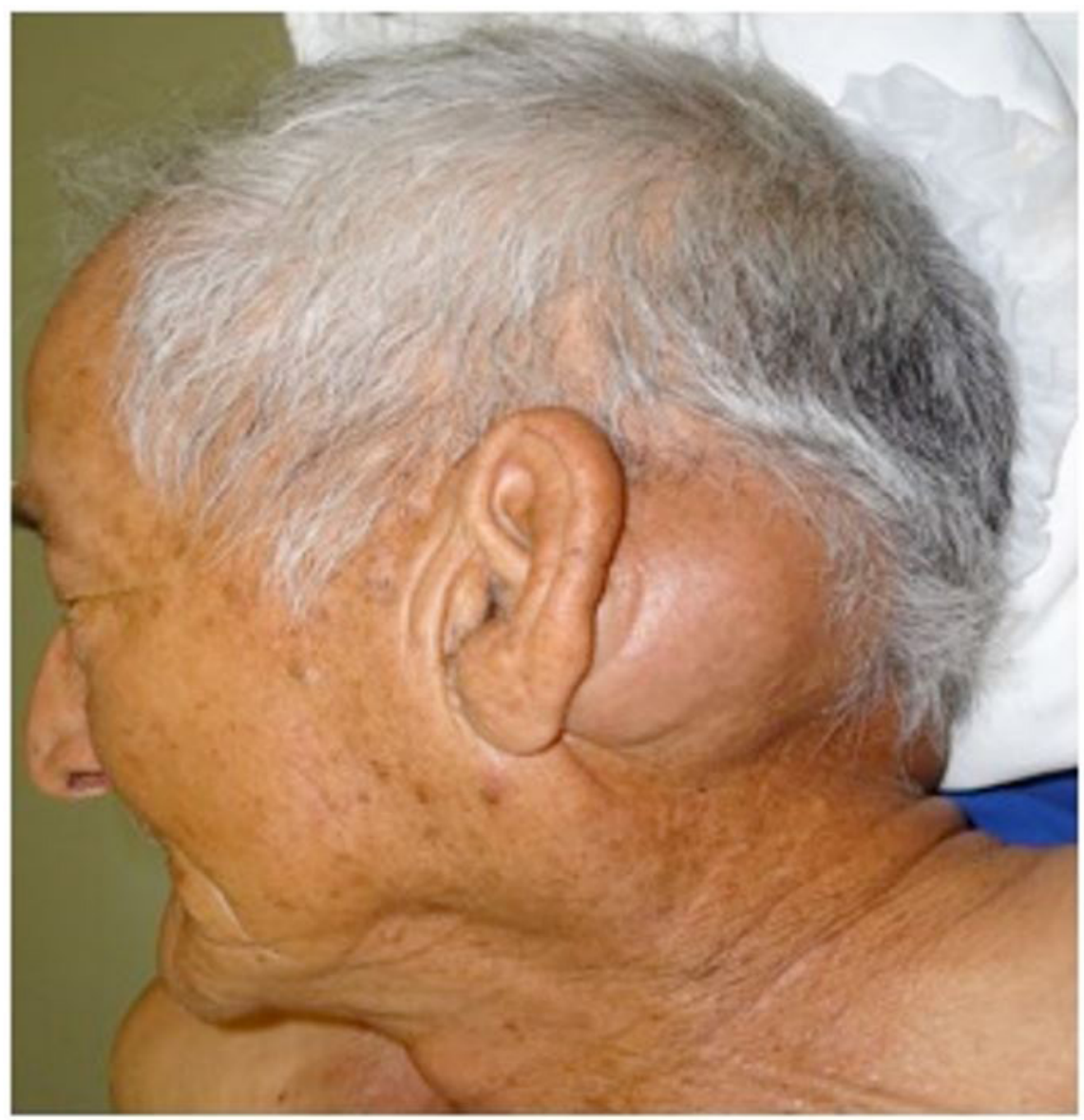

Figure 1. A 72-year-old male with a diagnosis of Kimura's disease. Tumor and edema are seen in the left retroauricular region. 


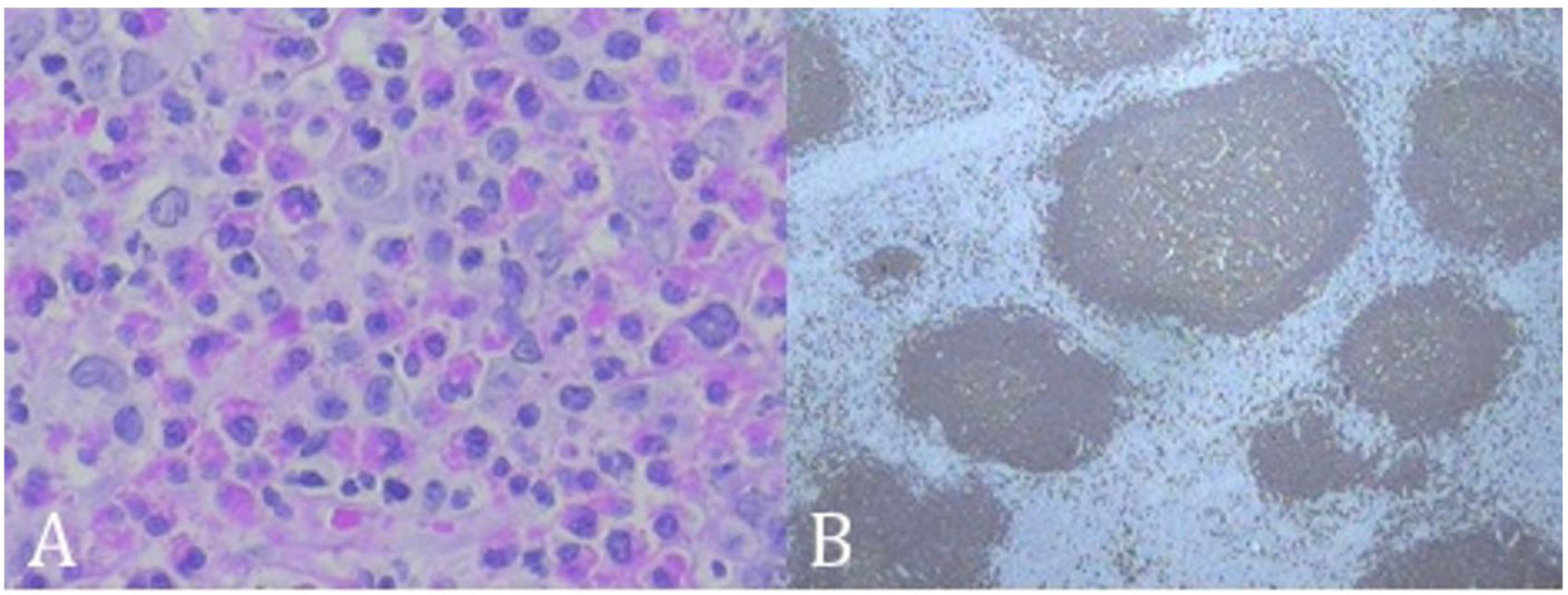

Figure 2. (A) Hematoxylin-Eosin A 100x increase demonstrates a marked inflammatory infiltrate associated with large numbers of eosinophils; (B) CD20 Increase 40x demonstrates a marking of the lymphocytes in the germinal centers evidencing a follicular hyperplasia.

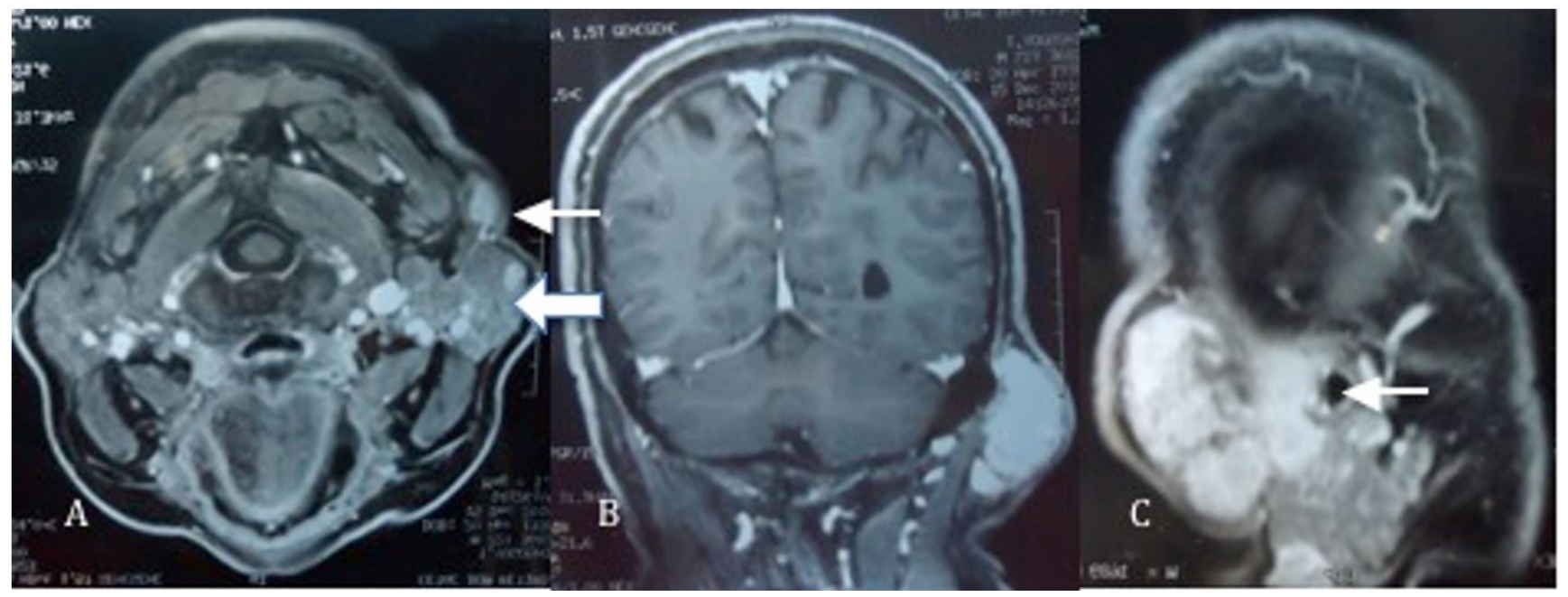

Figure 3. (A) T1 image demonstrating intermediate signal mass (thin arrow) without left parotid cleavage plane (thick arrow). There are some oval images characterized by an intermediate signal and with homogenous enhancement after contrast in the left parotid gland and also inside the gland, measuring up to about $1.4 \mathrm{~cm}$; (B) T2 image shows mass in left retroauricular intermediate signal; (C) T2 image shows mass in intermediate signal and its relation with external auditory canal (thin arrow).

\section{Discussions}

For many years, Kimura's disease was considered as a late stage of angiolymphoid hyperplasia with eosinophilia ${ }^{3}$. In 1948, Kimura et al. ${ }^{2}$ described KD as an uncommon granuloma associated with hyperplastic changes in lymphoid tissue. In 1987, Urable et al. and another author called Paul B. Googe in another article in the same year, described KD and angiolymphoid hyperplasia with eosinophilia as two different entities in clinical and pathological aspects 5 . In 1988, Kuo et al. confirmed the concept of KD as an independent pathological entity ${ }^{6}$. 
$\mathrm{KD}$ consists of a chronic inflammatory disease involving the dermis and the subcutaneous cellular tissue, characterized by one or multiple nodules or painless masses ${ }^{7}$. It occurs predominantly unilaterally in the head and neck region and it is associated with regional lymphadenopathy with or without involvement of the salivary glands, with the parotid gland more affected. The lesions may remain stable for years with the possibility of new lesions ${ }^{7}$.

The classical triad consists of painless subcutaneous mass (es) in the head and neck region, eosinophilia in peripheral blood and tissues, and marked increase in serum IgG (IgE) levels. It is more common in young patients, in the $3^{\text {rd }}$ decade, male $(2: 1)$ and Asian. Prevalence in patients of other races is considered low?

Histopathologically, it is characterized by exuberant follicular hyperplasia associated with an inflammatory lymphocytic infiltrate with formation of lymphoid follicles interspersed by eosinophilic aggregates and fibrosis, extending as a septa ${ }^{5,6}$. There are many blood vessels, however, these vessels are regular and well defined, unlike angiolymphoid hyperplasia with eosinophilia, which presents exuberant vascular proliferation with irregular and tortuous vessels $s^{5-7}$.

Differential diagnosis is also made with pathologies such as Hodgkin's lymphoma, non-Hodgkin's lymphoma, histiocytosis $X$, tuberculosis and other neoplastic and infectious diseases that manifest with lymph node enlargement in young people. The diagnosis of these entities occurs through microscopy and serology $y^{3,4}$.

The pathogenesis of KD is unknown and it is considered a systemic immune disorder. Eosinophilia and the increase in serum IgE levels allow KD to be considered a CD4 (+) T helper 2 (Th2) type allergic reaction ${ }^{3,4,7}$.

Treatment includes observation and follow-up in discrete and asymptomatic cases and surgical excision, which is the most common approach. Other less commonly used options include intralesional or systemic corticosteroids, cyclosporine, pentoxifylline, and radiation therapy. Post-surgery relapse occurs with a certain frequency ${ }^{4,7}$.

It is important the knowledge of Kimura's disease, as a possible diagnosis in the young patient, mainly of Asian origin, with painless cervical mass, associated with eosinophilia and hypergammaglobulinemia E.

\section{References}

1. Kim HT, Szeto C. Eosinophilic, hyperplastic lymphogranuloma, comparison with Mikulicz's disease. Chin Med J. 1937;23:699.

2. Kimura T, Yoshimura S, Ishikawa E. Unusual granulation combined with hyperplastic changes of lymphatic tissue. Trans Soc Pathol Jpn. 1948;37:179.

3. Chusid MJ, Rock AL, Sty JR, Oechler HW, Beste DJ. Kimuras disease: an unusual cause of cervical tumour. Arch Dis Child. 1997;77(2):153-4. http://dx.doi. org/10.1136/adc.77.2.153. PMid:9301357. 


\section{*Correspondence}

Paola Andrea Galbiatti Pedruzzi

Hospital Erasto Gaertner

Rua Dr. Ovande do Amaral, 201,

Cabral

CEP 80035-030, Curitiba (PR), Brasil

Tel.: +55 (41) 3361-5237

E-mail: paolapedruzzi@yahoo.com.br

\section{Authors information}

PAGP - MD; MSc; Head and Neck Surgery, Hospital Erasto Gaertner. BVO - MD; Head and Neck Surgery; Chief, Hospital Erasto Gaertner. JCLB - MD; Resident; Oncological Surgery, Hospital Erasto Gaertner. RTS - Dentist; Maxillofacial Surgery, Hospital Erasto Gaertner. AHW - MD; Resident, Pathology Department, Hospital Erasto Gaertner. JEJ - MD; PhD, Pathology Department, Hospital Erasto Gaertner.
4. Kung IT, Gibson JB, Bannatyne PM. Kimuras disease: a clinico-pathological study of 21 cases and its distinction from angiolymphoid hyperplasia with eosinophilia. Pathology. 1984;16(1):39-44. http://dx.doi.org/10.3109/00313028409067909. PMid:6718071.

5. Urabe A, Tsuneyoshi M, Enjoji M. Epithelioid hemangioma versus Kimura's disease: a comparative clinicopathologic study. Am J Surg Pathol. 1987;11(10):758-66. http://dx.doi.org/10.1097/00000478-198710000-00003. PMid:3116865.

6. Kuo T, Shih L-Y, Chan H-L. . Kimura's disease: involvement of regional lymph nodes and distinction from angiolymphoid hyperplasia with eosinophilia. Am J Surg Pathol. 1988;12(11):843-54. http://dx.doi.org/10.1097/00000478-19881100000005. PMid:2461103.

7. Yuen HW, Goh YH, Low WK, Lim-Tan SK. Kimuras disease: a diagnostic and therapeutic challenge. Singapore Med J. 2005;46(4):179-83. PMid:15800724. 